\title{
PIEPOC: A New Prognostic Index for Advanced Epithelial Ovarian cancer-Japan Multinational Trial Organisation OC01-01.
}

\author{
Satoshi Teramukai, Kazunori Ochiai, Harue Tada, Masanori Fukushima \\ Journal of Clinical Oncology August 1, 2007; 25 (22):3302-3306.
}

\section{SUMMARY:}

Ovarian cancer is the leading cause of death with highest fatality among all the gynecologic malignancies. Several factors are recognized as predictors of clinical outcome in patients with EOC. Stage and residual volume of tumour after primary surgical cytoreduction are the most consistently reported prognostic factors. ${ }^{1,2}$ Several studies have shown age, performance status (PS), histologic cell type, grade of the tumour and presence of ascites as independent prognostic factors. ${ }^{3}$

The present study was conducted by Japan Multinational Trial Organization with the aim of constructing a simple and powerful prognostic index (PI) of epithelial ovarian cancer ${ }^{4}$. Eight hundred and eighty women of stage III or IV epithelial ovarian cancer who were treated with adjuvant chemotherapy after maximal surgical debulking between 1994 and 2000 at 24 institutions in Japan were the participants of the study. 112 patients were excluded as information regarding PS, histologic cell type, and residual tumor size was not available. 768 patients were included in the study and evaluated for age, Eastern Cooperative Oncology Group PS, FIGO stage, histologic cell type, histologic grade, and residual tumour size. The patients were randomly split into a training sample for model validation and a validation sample to evaluate the reproducibility of the prognostic factor model. Survival curves were estimated using the
Kaplan- Meier method. The association between the prognostic factors and the overall survival were analyzed using the log-rank test. A Prognostic Index (PI) to predict the overall survival was developed by the proportional hazards regression model with backward elimination methods. Additivity of effects was checked by the pooled interaction test. Risk classification was developed based on Akaike's information criterion. Calibration curves of the relationship between the observed 5-year Kaplan-Meier estimates of overall survival and the predicted probabilities were examined for each group. Bootstrapping with 200 repetitions was used to obtain unbiased estimates. Concordance index was used to evaluate discrimination. Statistical analysis was done with SAS version 9.1.

Out of 768 patients, 408 had died at a median follow up of 4.1 years. $70 \%$ (538 patients) of all patients were selected as training sample in which independent prognostic factors were identified and a prognostic model was built. A simplified PI was derived as follows: PI $=1$ (if age 70 and above) +1 (if PS 1 or 2 ) +2 (if PS 3 or 4 ) +1 (if mucinous or clear-cell) +2 (if residual size $0.1 \mathrm{~cm}$ and above). The prognostic index classified patients into 3 risk groups: low, intermediate and high risk based on age, ECOG PS, histological cell type and residual tumour size which were analysed to be independently significant. 


\begin{tabular}{|l|l|l|l|}
\hline Risk Group & PI & $\begin{array}{l}\text { 5-year survival } \\
\text { Probability }\end{array}$ & Hazard Ratio (95\% CI) \\
\hline Low & $0-2$ & 0.67 & Reference group \\
\hline Intermediate & 3 & 0.43 & $2.29(1.44$ to 3.65$)$ \\
\hline High & $4-6$ & 0.17 & $4.87(2.97$ to 7.98$)$ \\
\hline
\end{tabular}

This could accurately predict 5-year survival in the validation sample. This was reproducible in all patients and in stage IIIC and IV patients as well. The PIEPOC was then calibrated to predict 5-year survival in all patients in both the training and validation samples. Predictive accuracy for 5-year survival considering individual PI and the 3 risk group approach was also maintained.

\section{COMMENTS}

The authors must be commended for a large study population and sound statistical analysis which has aided in the classification of patients into low, intermediate and high risk groups with respect to survival outcomes. Recently a retrospective review of data from 1895 patients treated in clinical trials conducted by all GOG protocols were pooled in aiming at identifying independent prognostic factors in this population. ${ }^{5}$ Age, PS, tumour histology and residual tumour volume were independent predictors of prognosis in patients with stage III EOC. Although the study population was large, all these factors are well associated with survival in previous studies and there was nothing novel in this GOG study. A Dutch study identified PS, residual tumour size, stage, histologic grade, and ascites as prognostic factors using data from two clinical trials. ${ }^{6}$ The first study that validated PI in patients with ovarian carcinoma was proposed by Lund et al. ${ }^{7}$ This study compared the PI of Dutch study and that of Danish study ${ }^{8}$ including PS, residual tumor size, age, and weight or body surface area from a clinical trial and proposed a final PI including information on PS and residual tumor size. The limitation of this study was, the classification method of risk groups according to the PI was not well specified.

The present study has some merits to consider which overcame the limitations of previous studies. The study population is large; prognostic-factor model is simple, long-term follow-up of the study and has sound statistical backup. They have done bootstrapping with 200 repetitions to obtain relatively unbiased estimates which is the strong point of this study. However, the heterogeneity of treatment using different chemotherapy regimes remains the limitation of the study. Paclitaxel and platinum combination, which is currently considered the standard treatment of epithelial ovarian cancer, was given only in $30 \%$ of the patients. The median survival of patients in this study is 49 months which is longer than that reported in studies from west. The applicability of this study to other populations is questioned in the study itself and needs further randomized controlled trials for its validation. This study also raises further question whether we have different treatment options to offer to different risk groups as the search for consolidation or maintenance therapy in ovarian cancer is still on!

\section{ACKNOWLEDGMENT}

We gratefully acknowledge help of Dr Guresh of Biostatistics Deptt. AIIMS for explaining the statistical part of this study. 


\section{REFERENCES:}

1. Hoskins WJ, Bundy BN, Thigpen T, et al: The influence of cytoreduction surgery on recurrence-free interval and survival in small-volume stage III epithelial ovarian cancer: A Gynecologic Oncology Group study. Gynecol Oncol 1992;47:159-166.

2. Ng LW, Rubin S, Hoskins WJ, et al: Aggressive chemosurgical debulking in patients with advanced ovarian cancer. Gynecol Oncol 1990;8:358-363.

Thigpen T, Brady MF, Omura GA, et al: Age as a prognostic factor in ovarian carcinoma. Cancer 1993;71:606-614.

4. Teramukai, S, Ochiai, K, Tada H, Fukushima $M$ PIEPOC: A New Prognostic Index for Advanced Epithelial Ovarian cancer-Japan Multinational Trial Organisation OC01-01. J Clin Oncol 2007;25(22):33023306.

5. Winter WE 3rd, Maxwell GL, Tian $C$ et al. Prognostic factors for stage III epithelial ovarian cancer: a
Gynecologic Oncology Group Study. J Clin Oncol. 2007;25(24):3621-7.

6. van Houwelingen JC, ten Bokkel Huinink $W W$, van der Burg MEL, et al: Predictability of the survival of patients with advanced ovarian cancer. $J$ Clin Oncol 1989;7:769-773.

7. Lund B, Williamson P, van Houwelingen HC, Neijt JP. Comparison of the predictive power of different prognostic indices for overall survival in patients with advanced ovarian carcinoma. Cancer Res. 1990; 50(15):4626-9

8. Lund, B., Hansen, M. Hansen. H. et al. A randomized study of sequential versus alternating combination chemotherapy in advanced ovarian carcinoma. Ann Oncol 1990;1(2):134-140.

Anjali Mookerjee, Meenakshi R, Roopa Hariprasad Department of Medical Oncology Dr. BRA Institute Rotary Cancer Hospital All India Institute of Medical Sciences, New Delhi-110029 